\title{
Cobaloximes with Mixed Dioximes of Glyoxime and Diphenylglyoxime: Synthesis, Characterization, CV, X-ray Studies and Crystal Packing
}

\author{
B. D. Gupta,* R. Yamuna and Debaprasad Mandal
}

Department of Chemistry, Indian Institute of Technology Kanpur, India 208016

Tel: +91-512-2597046; Fax: +91-512-2597436

\section{Supporting Information}

Table S1. Molar distribution

\begin{tabular}{|l|l|l|c|c|}
\hline Reactants & $\begin{array}{l}\text { RX } \\
\text { ClCo(gH)(dpgH)Py }\end{array}$ & $\begin{array}{l}\text { Comp } \\
\text { no }\end{array}$ & & \multicolumn{2}{|c|}{$\begin{array}{l}\text { \% molar distribution } \\
\text { (after column })\end{array}$} \\
\cline { 3 - 5 } & & & $\begin{array}{c}\text { a } \\
(\mathrm{dpgH})\end{array}$ & $\begin{array}{c}\text { b } \\
(\mathrm{gH})(\mathrm{dpgH})\end{array}$ \\
\hline Entry 1 & - & - & - & - \\
\hline Entry 2 & $\mathrm{NaBr}$ & 2 & 30 & 70 \\
\hline Entry 3 & $\mathrm{NaNO}_{2}$ & 3 & 10 & 90 \\
\hline Entry 4 & $\mathrm{NaN}_{3}$ & 4 & 9 & 91 \\
\hline Entry 5 & $\mathrm{MeI}$ & 5 & 27 & 73 \\
\hline Entry 6 & $\mathrm{EtI}$ & 6 & 13 & 87 \\
\hline Entry 7 & $\mathrm{PrBr}$ & 7 & & \\
\hline Entry 8 & $\mathrm{BuBr}$ & 8 & 27 & 73 \\
\hline Entry 9 & $\mathrm{PentBr}$ & 9 & 37 & 62 \\
\hline Entry 10 & $\mathrm{HexBr}$ & 10 & 31 & 69 \\
\hline Entry 11 & $\mathrm{HeptBr}$ & 11 & 34 & 66 \\
\hline Entry 12 & $\mathrm{OctBr}$ & 12 & 36 & 64 \\
\hline Entry 13 & $\mathrm{NonBr}$ & 13 & 17 & 83 \\
\hline Entry 14 & $\mathrm{DecBr}$ & 14 & 36 & 64 \\
\hline
\end{tabular}


Table S2. Co-ordination shift* from ${ }^{13} \mathrm{C}$ and ${ }^{1} \mathrm{H}$ NMR values for $\mathbf{1 b}-\mathbf{1 4 b}$

\begin{tabular}{|l|l|l|l|l|l|l|}
\hline No & $\Delta \delta^{13} \mathrm{CPy}_{\alpha}$ & $\Delta \delta^{13} \mathrm{CPy}_{\beta}$ & $\Delta \delta^{13} \mathrm{CPy}_{\gamma}$ & $\Delta \delta^{1} \mathrm{H} \mathrm{Py}_{\alpha}$ & $\Delta \delta^{1} \mathrm{H} \mathrm{Py}_{\beta}$ & $\Delta \delta^{1} \mathrm{H} \mathrm{Py}_{\gamma}$ \\
\hline 1b & 1.00 & 2.42 & 3.65 & -0.16 & 0.29 & 0.38 \\
\hline 2b & 0.72 & 2.43 & 3.55 & -0.16 & 0.31 & 0.38 \\
\hline 3b & 0.53 & 2.41 & 3.68 & -0.11 & 0.33 & 0.41 \\
\hline 4b & 1.16 & 2.49 & 3.61 & -0.13 & 0.31 & 0.39 \\
\hline 5b & 0.03 & 1.89 & 2.16 & 0.20 & 0.38 & 0.40 \\
\hline 6b & -0.01 & 1.87 & 2.15 & 0.19 & 0.37 & 0.38 \\
\hline 7b & 0.03 & 1.84 & 2.07 & 0.19 & 0.36 & 0.38 \\
\hline 8b & 0.04 & 1.84 & 2.07 & 0.19 & 0.36 & 0.38 \\
\hline 9b & 0.04 & 1.84 & 2.05 & 0.19 & 0.36 & 0.38 \\
\hline 10b & 0.05 & 1.83 & 2.05 & 0.20 & 0.36 & 0.38 \\
\hline 11b & 0.07 & 1.84 & 2.05 & 0.19 & 0.36 & 0.38 \\
\hline 12b & 0.05 & 1.84 & 2.05 & 0.19 & 0.36 & 0.38 \\
\hline 13b & 0.04 & 1.83 & 2.05 & 0.19 & 0.36 & 0.38 \\
\hline 14b & 0.04 & 1.84 & 2.06 & 0.19 & 0.36 & 0.38 \\
\hline
\end{tabular}

${ }^{* 1} \mathrm{H}$ of free Py: $\alpha=8.57 ; \beta=7.05 ; \gamma=7.43$ ppm: ${ }^{13} \mathrm{C}$ of free Py: $\alpha=149.82 ; \beta=123.73 ; \gamma=$ $135.89 \mathrm{ppm}$. 
Table S3. Effect of one wing on the other wing $\left({ }^{13} \mathrm{C} N M R\right)$ for $\mathbf{1 b}-\mathbf{1 4 b}$

\begin{tabular}{|l|l|l|l|l|}
\hline No & $\begin{array}{l}\delta_{\mathrm{C}=\mathrm{N}} \\
(\mathrm{dpgH})\end{array}$ & No & $\begin{array}{l}\delta_{\mathrm{C}=\mathrm{N}} \\
(\mathrm{dpgH} \\
\text { wing })\end{array}$ & $\Delta_{\mathrm{dpgH}}$ \\
\hline 1a & 153.58 & 1b & 154.02 & 0.44 \\
\hline 2a & 154.05 & 2b & 154.56 & 0.51 \\
\hline 3a & 153.72 & 3b & 153.99 & 0.27 \\
\hline 4a & 153.34 & 4b & 153.87 & 0.53 \\
\hline 5a & 150.77 & 5b & 151.21 & 0.44 \\
\hline 6a & 150.79 & 6b & 151.22 & 0.43 \\
\hline 7a & 150.84 & 7b & 151.33 & 0.49 \\
\hline 8a & 150.80 & 8b & 151.28 & 0.48 \\
\hline 9a & 150.81 & 9b & 151.26 & 0.45 \\
\hline 10a & 150.79 & 10b & 151.27 & 0.48 \\
\hline 11a & 150.80 & 11b & 151.26 & 0.46 \\
\hline 12a & 150.78 & 12b & 151.26 & 0.48 \\
\hline 13a & 150.76 & 13b & 151.26 & 0.50 \\
\hline 14a & 150.77 & 14b & 151.26 & 0.49 \\
\hline
\end{tabular}

$\Delta_{\mathrm{dpgH}}={ }^{13} \mathrm{C} \delta_{1 \mathrm{~b}-14 \mathrm{~b}^{-}}{ }^{13} \mathrm{C} \delta_{1 \mathrm{a}-14 \mathrm{a} \text { (corresponding values) }}$ (effect of $\mathrm{gH}$ wing over dpgH)

Table S4. Effect of one wing on the other wing $\left({ }^{13} \mathrm{C}\right.$ NMR) for $\mathbf{1 b}-\mathbf{1 4 b}$

\begin{tabular}{|l|l|l|l|l|}
\hline No & $\begin{array}{l}\delta_{\mathrm{C}=\mathrm{N}} \\
(\mathrm{gH})\end{array}$ & No & $\begin{array}{l}\delta_{\mathrm{C}=\mathrm{N}} \\
(\mathrm{gH} \text { wing })\end{array}$ & $\Delta_{\mathrm{gH}}$ \\
\hline $\mathrm{ClCo}(\mathrm{gH})_{2} \mathrm{Py}(\mathbf{1 c})$ & 140.56 & $\mathbf{1 b}$ & 140.28 & -0.28 \\
\hline $\mathrm{BrCo}(\mathrm{gH})_{2} \mathrm{Py}(\mathbf{2 c})$ & 141.10 & $\mathbf{2 b}$ & 140.72 & -0.38 \\
\hline $\mathrm{NO}_{2} \mathrm{Co}(\mathrm{gH})_{2} \mathrm{Py}(\mathbf{3 c})$ & 140.48 & $\mathbf{3 b}$ & 140.33 & -0.15 \\
\hline $\mathrm{N}_{3} \mathrm{Co}(\mathrm{gH})_{2} \mathrm{Py}(\mathbf{4 c})$ & 139.85 & $\mathbf{4 b}$ & 139.81 & -0.04 \\
\hline $\mathrm{CH}_{3} \mathrm{Co}(\mathrm{gH})_{2} \mathrm{Py}(\mathbf{5 c})$ & 137.94 & $\mathbf{5 b}$ & 137.73 & -0.21 \\
\hline $\mathrm{C}_{2} \mathrm{H}_{5} \mathrm{Co}(\mathrm{gH})_{2} \mathrm{Py}(\mathbf{6 c})$ & 137.95 & $\mathbf{6 b}$ & 137.82 & -0.13 \\
\hline $\mathrm{C}_{3} \mathrm{H}_{7} \mathrm{Co}(\mathrm{gH})_{2} \mathrm{Py}(\mathbf{7 c})$ & 138.03 & $\mathbf{7 b}$ & 137.85 & -0.18 \\
\hline $\mathrm{C}_{4} \mathrm{H}_{9} \mathrm{Co}(\mathrm{gH})_{2} \mathrm{Py}(\mathbf{8 c})$ & 137.97 & $\mathbf{8 b}$ & 137.81 & -0.16 \\
\hline $\mathrm{C}_{5} \mathrm{H}_{11} \mathrm{Co}(\mathrm{gH})_{2} \mathrm{Py}(\mathbf{9 c})$ & 137.96 & $\mathbf{9 b}$ & 137.80 & -0.16 \\
\hline $\mathrm{C}_{6} \mathrm{H}_{13} \mathrm{Co}(\mathrm{gH})_{2} \mathrm{Py}(\mathbf{1 0 c})$ & 138.01 & $\mathbf{1 0 b}$ & 137.79 & -0.22 \\
\hline $\mathrm{C}_{7} \mathrm{H}_{15} \mathrm{Co}(\mathrm{gH})_{2} \mathrm{Py}(\mathbf{1 1 c})$ & 138.02 & $\mathbf{1 1 b}$ & 137.80 & -0.22 \\
\hline $\mathrm{C}_{8} \mathrm{H}_{17} \mathrm{Co}(\mathrm{gH})_{2} \mathrm{Py}(\mathbf{1 2 c})$ & 137.95 & $\mathbf{1 2 b}$ & 137.79 & -0.16 \\
\hline $\mathrm{C}_{9} \mathrm{H}_{19} \mathrm{Co}(\mathrm{gH})_{2} \mathrm{Py}(\mathbf{1 3 c})$ & 138.02 & $\mathbf{1 3 b}$ & 137.80 & -0.22 \\
\hline $\mathrm{C}_{10} \mathrm{H}_{21} \mathrm{Co}(\mathrm{gH})_{2} \mathrm{Py}(\mathbf{1 4 c})$ & 138.04 & $\mathbf{1 4 b}$ & 137.80 & -0.24 \\
\hline
\end{tabular}

$\Delta_{\mathrm{gH}}={ }^{13} \mathrm{C} \delta_{1 \mathrm{~b}-14 \mathrm{~b}}{ }^{13} \mathrm{C} \delta_{1 \mathrm{c}-14 \mathrm{c} \text { (corresponding values) }}$ (effect of dpgH wing over $\mathrm{gH}$ ) 


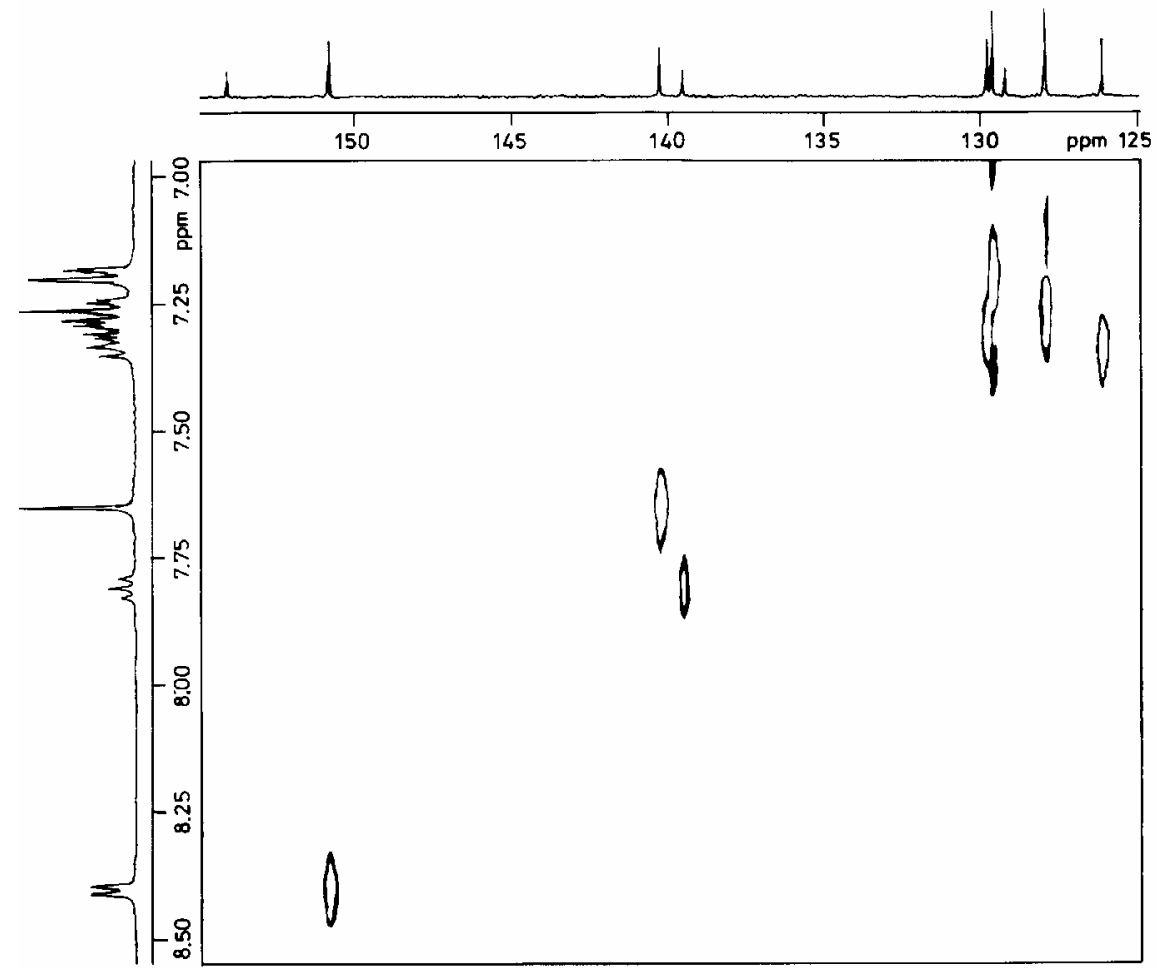

Figure S1 ${ }^{1} \mathrm{H}-{ }^{13} \mathrm{C}$ correlation NMR spectrum of $\mathrm{ClCo}(\mathrm{gH})(\operatorname{dpgH}) \mathrm{Py}(\mathbf{1 b})$

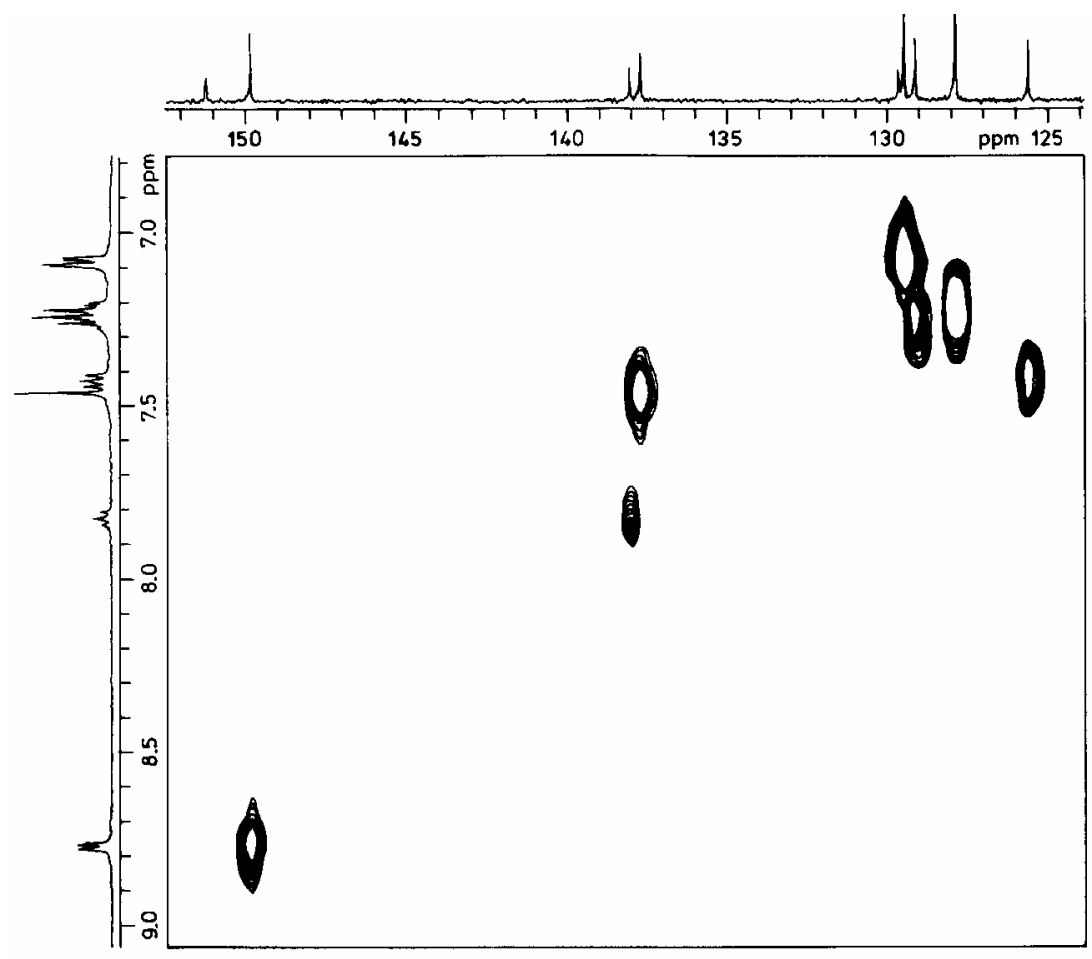

Figure S2 ${ }^{1} \mathrm{H}-{ }^{13} \mathrm{C}$ correlation NMR spectrum of $\mathrm{MeCo}(\mathrm{gH})(\mathrm{dpgH}) \mathrm{Py}(\mathbf{5 b})$ 


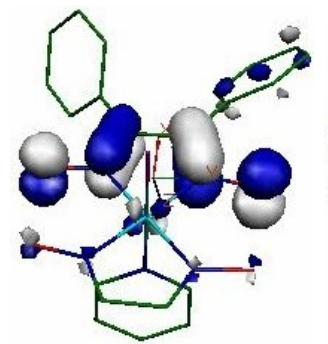

122

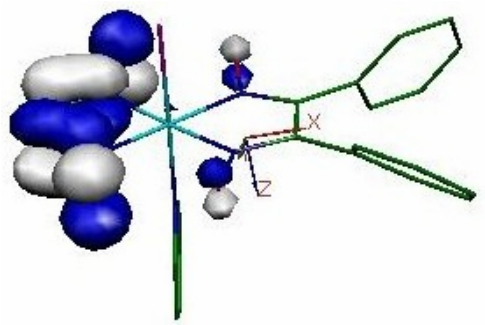

123 (HOMO)

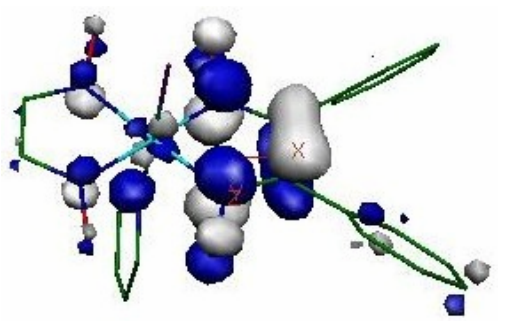

124(LUMO)

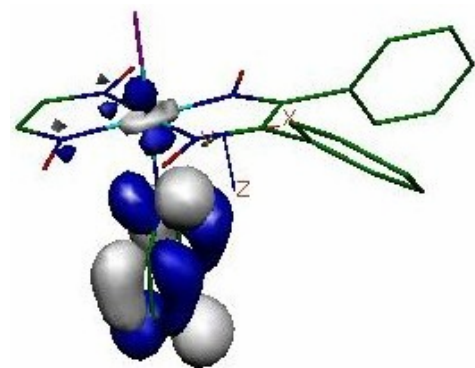

125

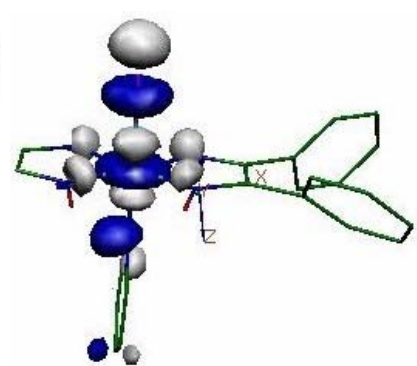

126

HOMO - responsible for reduction potentials

LUMO - responsible for oxidation potentials

Figure S3. MO picture of $\mathrm{ClCo}(\mathrm{gH})(\mathrm{dpgH}) \mathrm{Py}(\mathbf{1 b})$ from ab inito calculation by DFT

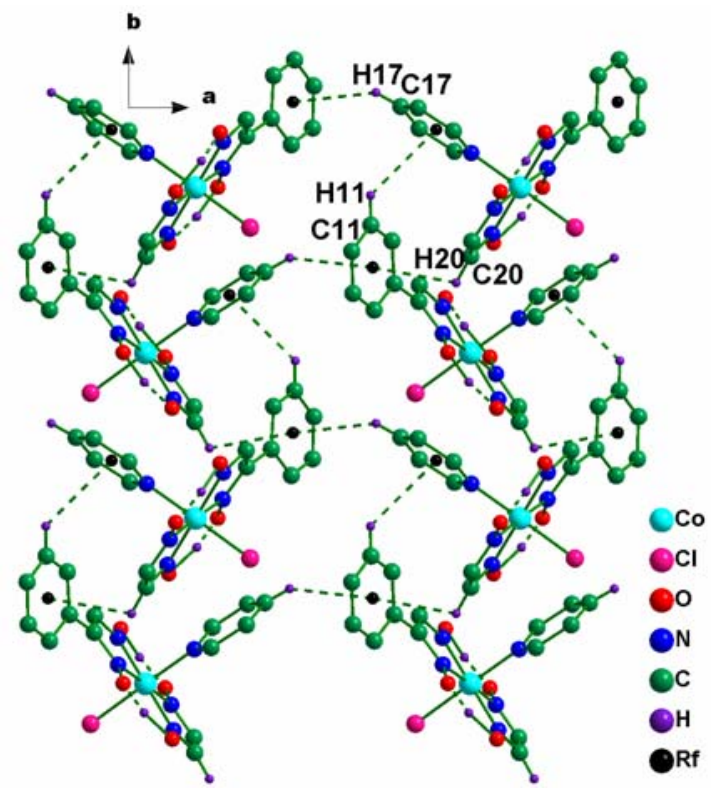

Figure S4. 2-D helical assembly of $\mathbf{1 b}$ along the c-axis. One of the phenyl ring and all the hydrogen atoms except those involved in hydrogen bonding have been omitted for clarity. 


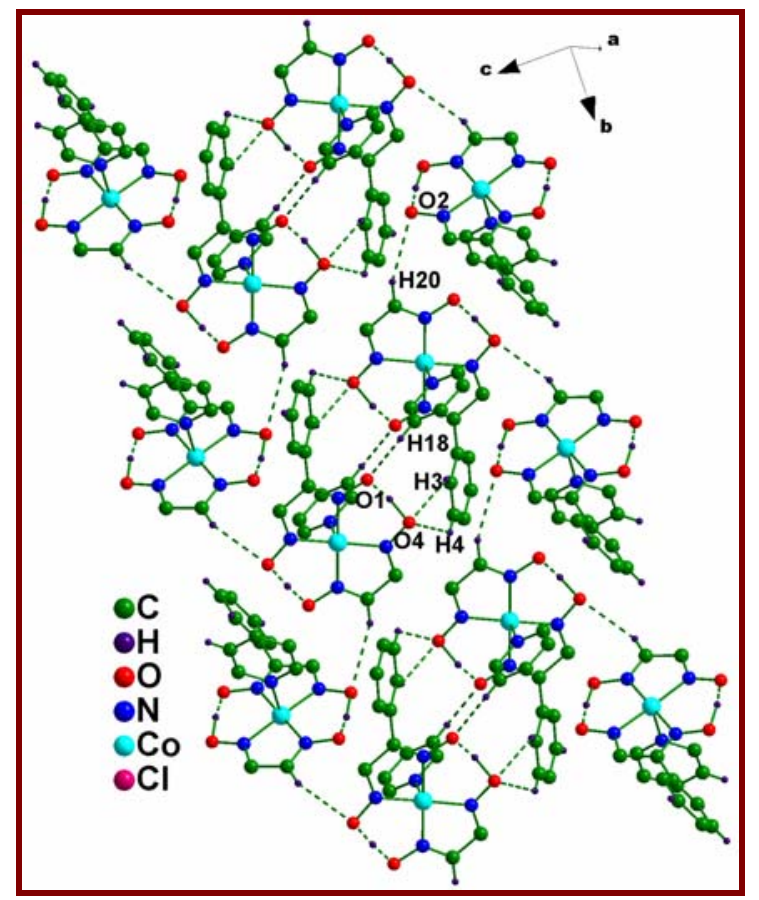

Figure S5. C-H $\cdots$ O bond assisted supramolecular 2-D lamellar structure of $\mathbf{1 b}$ (view along the a-axis). One of the phenyl ring and most of the hydrogen atoms are not shown for clarity. 


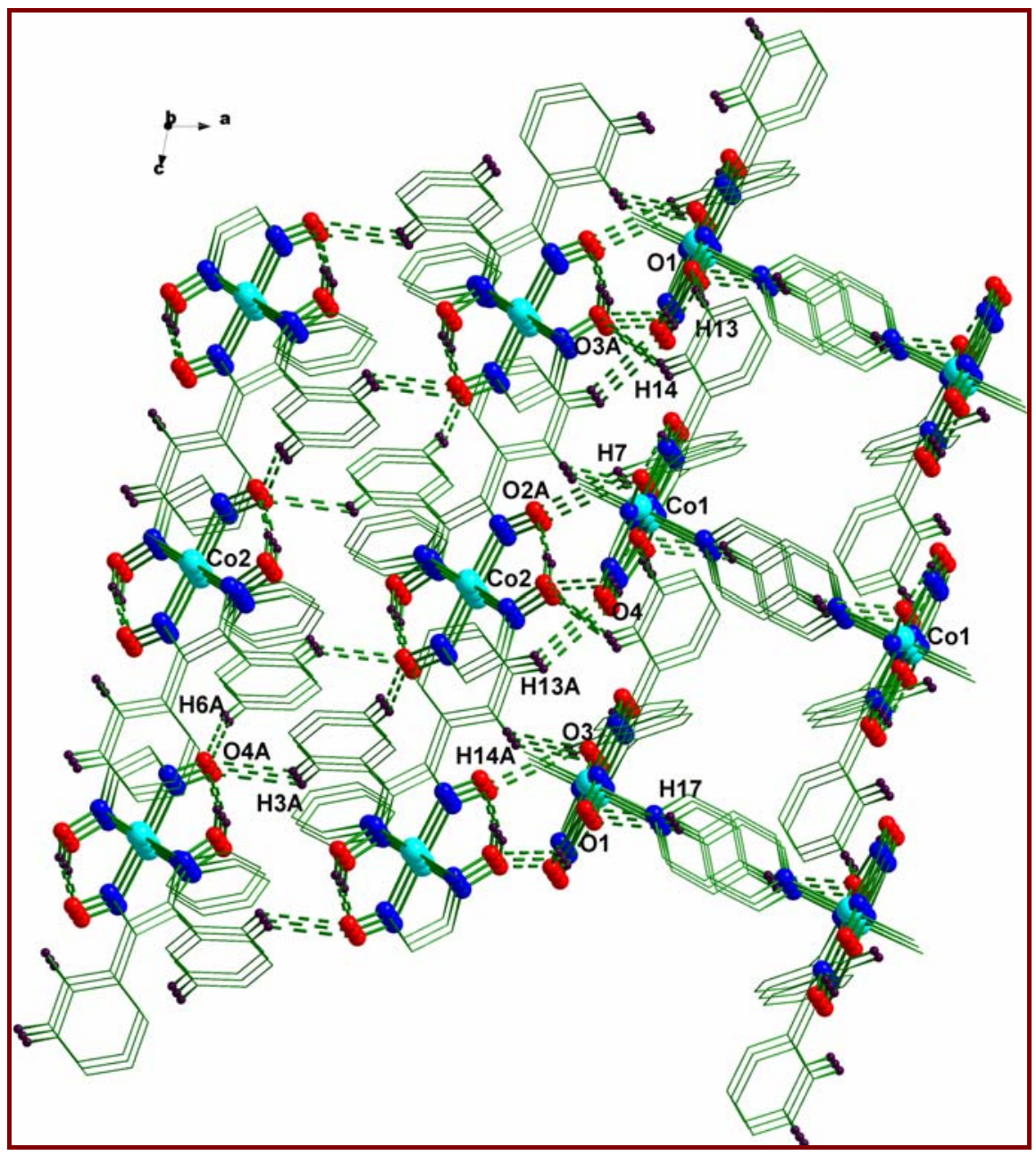

Figure S6. 3-D network of 6b-I and 6b-II (view along the b-axis). One of the methyl group of $\mathrm{R}$ and all the hydrogen atoms except those involved in hydrogen bonding have been omitted for clarity. 


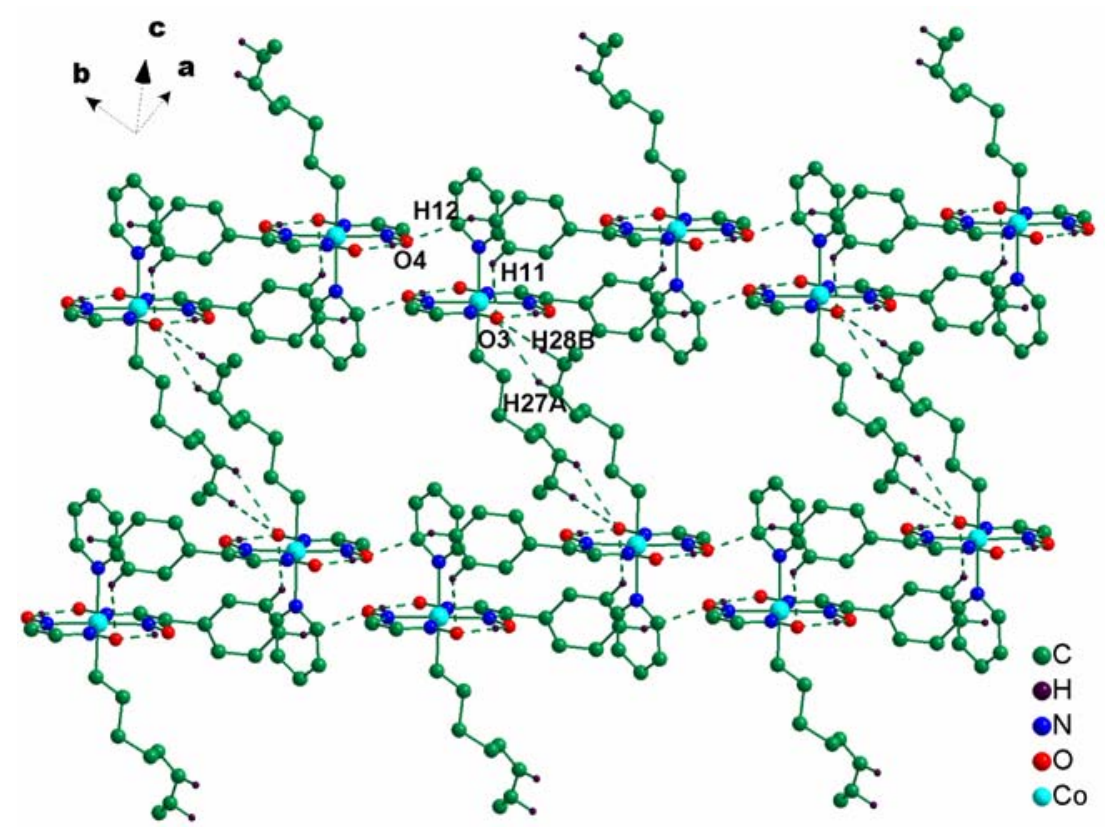

Figure S7. 2-D lamellar network of $\mathbf{1 2 b}$ (projection down the c-axis). One of the phenyl ring, and all the hydrogen atoms except those involved in hydrogen bonding have been omitted for clarity. 